\title{
MODELLING COLLABORATIVE SERVICES The COSEMO Model
}

\author{
Thanh Thoa Pham Thi ${ }^{1}$, Thang Le Dinh ${ }^{2}$ \\ ${ }^{I}$ School of Computing, Dublin City University, Glasnevin, Dublin 9, Ireland \\ ${ }^{2}$ Université du Québec à Trois-Rivières, Trois-Rivières, Quebec, Canada \\ Markus Helfert ${ }^{3}$, Michel Leonard ${ }^{4}$ \\ ${ }^{3}$ School of Computing, Dublin City University, Glasnevin, Dublin 9, Ireland \\ ${ }^{4}$ Department of Information Systems, University of Geneva, Switzerland
}

Keywords: Service Modelling, Collaboration, Sharing Data and Processes, Collaborative Services.

\begin{abstract}
Despite the dominance of the service sector in the last decades, there is still a need for a strong foundation on service design and innovation. Little attention has been paid on service modelling, particularly in the collaboration context. Collaboration is considered as one of the solutions for surviving or sustaining the business in the high competitive atmosphere. Collaborative services require various service providers working together according to agreements between them, along with service consumers, in order to coproduce services. In this paper, we address crucial issues in collaborative services such as collaboration levels, sharing data and processes due to business interdependencies between service stakeholders. Subsequently, we propose a model for Collaborative Service Modelling - the COSEMO model, which is able to cover identified issues. We also apply our proposed model to modelling an example of Travelling services in order to illustrate the relevance of our modelling approach to the matter in hand.
\end{abstract}

\section{INTRODUCTION}

Service science has emerged in the last few years as an interdisciplinary research domain that addresses challenges in service innovation in the service sector. The service sector includes all economic activities whose output is not a tangible product and is generally consumed at the same time it is produced and provides added value in intangible forms (Quinn et al., 1987). Today, more and more business organizations have been seeking collaborations as one amongst solutions to sustain their business in high competitive environments. For instance, they use the supply chain model or alliance model. In such context, collaborative service is a kind of business collaboration in the service sector in which several business organizations work together to co-produce services.

Although the dominance of the service sector in recent years, a strong foundation on service design and innovation is still needed (Bitner et al, 2008). Service modelling is undertaken in the service design and innovation. Service modelling amounts to the representation of relations between what is provided to customers, how it is provided, the technical definition of the service, and resources needed for operating the service (Vilho Raisanen, 2006). Therefore at the informational level, service modelling should describe the creation and transformation of information between service stakeholders.

Due to data and process sharing in the collaboration context, some critical issues have emerged, such as various data sharing levels collaboration levels, and the consistency of data and process sharing across organizations to ensure correct performing of business activities.

Current approaches for service modelling often focus on single services. There are few approaches working on inter-organizational business process collaboration. However, they do not cover issues mentioned. In this paper, we propose our approach for collaborative service modeling as a solution to the matter in hand. 
The rest of the paper is organized as follows: Section 2 dues with related work in service and collaborative service modelling. Section 3 brings out crucial challenges in collaborative services and presents our modelling concepts that address these challenges. Finally, we conclude our work and give some research directions in Section 4.

\section{RELATED WORK}

Services are defined as "the application of competences for the benefit of another, meaning that service is a kind of action, performance or promise that is exchanged for value between provider and client" (Spohrer, 2007).

In our viewpoint, collaborative services are services created based on collaborations of service co-providers. For instance, we take an example of Travelling service for the illustration. A hotel collaborates with a travel agency who provides booking services to customers. Customers can contact the travel agency for their booking or cancellation, or they can also book or cancel their reservation directly with the hotel. The hotel also collaborates with a cleaning agency for the cleaning service.

In literature, there are some approaches to modelling single services which deals with only one service provider and service consumer(s) such as Molecular model, and Blueprinting approach.

Molecular model is one of the earliest models for service and product modelling developed by Shostack (Shostack, 1982). The centre of molecular model describes core benefit provided to customers which includes service elements, product elements, relationships between elements, and service evidences. These elements can be visualised with graphical notations. Other elements such as price strategy, distribution strategy, and advertisement strategy, etc. are layers outside the core of molecular describing the total market entity.

The blueprinting is also developed by Shostack (Shostack, 1984) and then evolved by (KingmanBrundage, 1995), (Vilho Räisänen, 2006), (Zeithaml, 2008) which has focused on processes that constitute the service. A service blueprint is a two-dimensional diagram. The horizontal axis represents the chronology of processes or functions in the service. The vertical axis represents different processes areas.

Recently, the Business Process Modelling Notation (BPMI, 2004) has been adapted for collaborative business process modelling for the
Service Oriented Architecture design (Touzi et al, 2009).

Similar, (Grossman, 2008) has proposed some extensions to business process modelling languages which enable them to describe various types of interprocess dependencies across organizations. They are for example, Triggering dependencies, Enabling dependencies, Cancelling dependencies, Disabling dependencies.

In the Service Oriented Computing paradigm, service composite and orchestration describe aggregating multiple services into a single composite service (Papazoglou et al. 2007). The composite service is often modelled with Petri Nets (Gehlot, Edupuganti 2009; Yoo et al. 2010).

These approaches have some limitations on describing various collaboration degrees, monitoring data consistency and monitoring shared processes performed across organizations.

\section{ISSUES IN MODELLING COLLABORATIVE SERVICES}

In this section firstly we present our meta modelling concepts, then we address crucial issues in collaborative services modelling and illustrate how to overcome it with our concepts.

\subsection{Modelling Concepts - The COSEMO Model}

We focus on data and process sharing between organizations during the production of services. The relevant concepts are as follows:

- Role describes organizational units inside/outside an organization or participants in the collaboration context

- Process is a business activity, a task or function

-Class represents information/data created/ transferred/transformed by process.

- Privileges on data: a role can have privileges of creation on data (i.e. class), and privilege of suppression, modification, reference on their own data (data created by this role). Moreover, a role can have privileges of creation, suppression, and reference on data created by other roles. In this case, they are denoted by suppression+, modification+, and reference +

- Responsibility on process: a role has responsibility on the performing of a process

- Available/Unavailable data: data in a class can be 
available or unavailable to certain processes in certain times (i.e. is able to participate in the process or is not). This often concerns data status changes. For example a booking is available to the Cancel and Confirm processes. If the booking becomes cancelled (after performing cancel process), then it is becomes unavailable to Cancel and Confirm process. But a confirmed booking is available to Cancel process.

In the following sections we will describe challenges in collaborative service modelling and illustrate with our concepts.

\subsection{Collaboration Level Modelling}

The collaboration level between organizations/ service providers is described by the degree of sharing data and process. There are four collaboration levels:

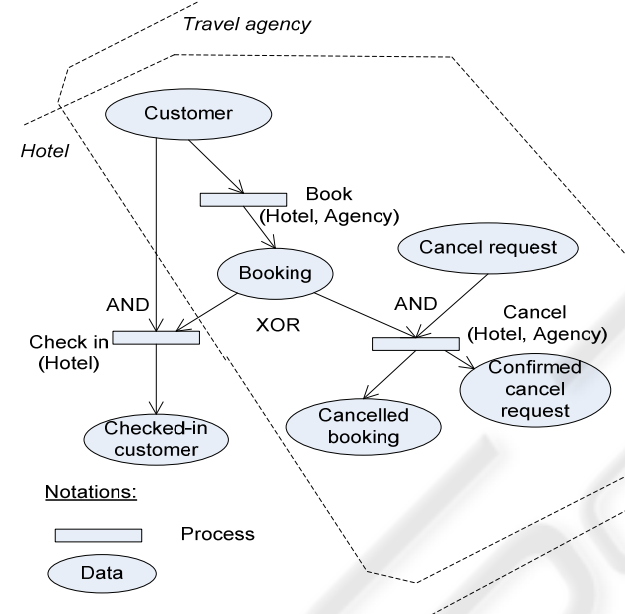

Figure 1: Very tight collaboration

Level 1 - very tight collaboration-data and process sharing, it is allowed to modify the shared data.

Figure 1 illustrates this collaboration level with the Travelling example. There are shared information and common processes between the hotel and the travel agency.

If a booking is made with the hotel then the customer change booking information or cancel it within the hotel. But if a booking is made with the travel agency, then the customer can change booking information or cancel it within the travel agency or the hotel. Customer information created/owned by the travel agency should be shared with the hotel, but the hotel does not need to share information with the Customer who directly booked with the hotel (i.e. the hotel has the privilege reference+ on
Customer, but the travel agency just has the privilege reference on Customer). The hotel can modify Booking shared by the travel agency, but it is not an inverse case (i.e. the hotel has privilege modification + on Booking, and the travel agency just has privilege modification on Booking), and so on.

Level 2 - tight collaboration- data and process sharing, it is not allowed to modify the share data.

For instance, concerning travelling service, an alliance of airlines shares the process of Bonus Calculation for loyalty clients to each member. The shared information between them for the performing of this process is information on flights taken, number of miles taken and concerning customer. However, an airline can not modify this shared information which is owned/created by other airlines.

Level 3 - loose collaboration: actively data sharing, it is not allowed to modify the shared data.

This case often concerns requesting other service providers to carry out a business activity/process. Figure 2 illustrates this level. For example, the hotel shares occupied room data to the cleaning agency for the cleaning service. Cleaning service just has the only privilege of reference on occupied room.

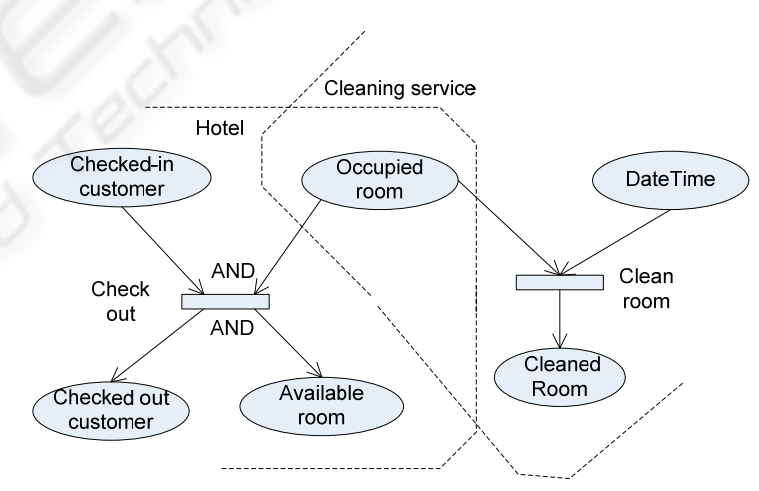

Figure 2: Loose collaboration

Level 4 - very loose collaboration: passively data sharing, it is not allowed to modify shared data.

A service provider passively shares some information to other service provider for the reference purpose, the shared information is not allowed to modify. The receiver just has the privilege of reference on shared data.

\subsection{Data and Process Consistency Control}

Consistency issues emerged due to data and process interdependencies among service providers. 
Figure 1 describes an exclusive constraint between Check-in process and Cancel process which span across organizations. Figure 2 describes a more complex situation which means "for every room, if the check-out process is performed then the clean room process is disabled, but it is not the case on the contrary".

We uses the concept of Available/Unavailable data in our model to solve this issue. In the first case, Booking becomes unavailable to the Cancel process and Check-in process once it participated in one of these processes. Therefore, if a booking is cancelled, later it can be checked-in, or vice-versa. Meanwhile in the second case, Occupied room is always available to the Clean room process, therefore an occupied room after cleaning is still occupied, until the Check-out process is performed.

\section{CONCLUSIONS}

Researchers have contributed some work on collaborative business process modelling, composite service and orchestration modelling. However, in this paper we showed that there are still some limitations on describing critical issues in collaborative service modelling which concerns the description of collaboration levels, data and process consistency across organizations due to data and process sharing needs. By addressing these issues, we proposed our meta-modelling concepts, the COSEMO model. This modelling approach is business oriented, not a formalism oriented approach like Petri Nets, therefore our approach avoids complex transformation of modelling due to constraints on formalisms.

Our future research directions will address the need of investigation of customer-centric characteristics of services. This allows the service customization and adaptation of customers according to their needs. The the modelling should be an adaptable approach which facilitates evolution.

\section{REFERENCES}

Bitner, M. J., Ostrom, A. L., Morgan F. N., 2008. Service Blueprinting: A Practical Technique for Service Innovation. In: California Management Review, Vol. 53, No. 3, pp.66--94.

Business Process Management Initiative (BPMI), 2004. Business Process Modeling Notation, Version1.0.

Grossmann G., Schrefl M., Stumptner M., 2008. Modelling Inter-Process Dependencies with High
Level Business Process Modelling Languages. In Proc.5th Asia-Pacific Conference on Conceptual Modelling, Wollongong, Australia.

Kingman-Brundage, J., George, W. R, 1995. Service logic: achieving service system integration. In International Journal of Service Industry Management, 6 (4), 20-39.

Kosanke K., 2005. ISO standards for interoperability: a comparison. In: Proceedings of the First International Conference on Interoperability of Enterprise Software and Applications INTEROP-ESA'05, Springer, Berlin, pp.55-64, ISBN 1-84628-151-2.

Papazoglou M.P., Traverso P., Dustdar S., Leymann F. 2007. "Service-Oriented Computing: State of the Art and Research Challenges", IEEE Computer, November, pp. 64-71.

Quinn, J. B., Baruch, J. J., and Paquette, P. C., 1987. "Technology in Services", Scientific American, Vol. 257, No. 6, pp.50-58.

Shostack, L. G., 1984. Design Services that Deliver. In Harvard Business Review (84115), 133-139.

Shostack, L. G., 1982. How to Design a Service. In European Journal of Marketing, 16(1), 49-63.

Shostack, L. G., 1987. Service Positioning Through Structural Change, Journal of Marketing, pp.34-43.

Spohrer, J., Maglio, Paul P., Bailey, J., Gruhl, D., 2007. Steps Towards a Science of Service Systems. In IEEE Computer, No.1, pp.71-77.

Touzi J., Benaben F., Pingaud H., Lorre J. P., 2009. A model-driven approach for collaborative serviceoriented architecture design. In International Journal Production Economics, Elsevier Publisher, 121, pp.520

Vilho Räisänen, 2006. Service Modelling: Principles and Applications. Wiley.

Yoo T., Jeong B., Cho H. 2009. "A Petri Nets based functional validation for services composition", in Journal of Expert Systems with Applications, Vol. 37, pp. $3768-3776$

Zeithaml, V., Bitner, M. J., Gremler, D., 2008. Services Marketing, Irwin Mcgraw-Hill (2000) 22. 